\title{
The Norwegian National Reporting System and Register of Severe Allergic Reactions to Food
}

\author{
Martinus Løvik ${ }^{1}$, Ellen Namork ${ }^{1}$, Christiane Fæste ${ }^{2}$ and Eliann Egaas ${ }^{2}$ \\ 1) Department of Environmental Immunology, Division of Environmental Medicine, \\ Norwegian Institute of Public Health, Oslo, Norway \\ 2) National Veterinary Institute, Oslo, Norway \\ Correspondence: Martinus Løvik, Department of Environmental Immunology, P.O. Box 4404 Nydalen, NO-0403 Oslo, Norway \\ Telephone: +4722042664/91377388 Telefax: +4722042686 E-mail: martinus.lovik@fhi.no
}

\begin{abstract}
SUMMARY
The Norwegian National Reporting System and Register of Severe Allergic Reactions to Food, or the Food Allergy Register, is a nation-wide, government-funded permanent reporting and registration system for severe allergic reactions to food. The Food Allergy Register collects information based on a one-page reporting form, serum samples for specific IgE analysis, and food samples for food allergen analysis. Reporting physicians receive in return an extensive commentary on the reported case and the relevant allergies, and results of the specific IgE analysis and food allergen analysis.

The Food Allergy Register has, after being active for a little more than four years, given valuable information about several important aspects of food allergy in Norway. The Food Allergy Register has revealed food safety problems in relation to allergy that probably could be discovered only with the help of a systematic, nation-wide registration of cases. The reactions of peanut allergic individuals to lupine flour in bakery products is an example of how the Food Allergy Register is able to reveal potentially serious problems that would otherwise probably have gone unnoticed and certainly unexplained. The amount and the value of the information from the Food Allergy Register are increasing as new reports of more cases are added. The typical Norwegian patient with a severe allergic reaction to food appears to be a young adult, female rather than male. The offending meal is consumed at a restaurant or fast-food stand or in a private party away from home, and peanuts, nuts and shellfish are among the most common offending foods, while fish allergy appears to be rather rare.
\end{abstract}

\section{INTRODUCTION}

According to public perception, allergy to foods and food additives represents a major health problem. However, there is a huge gap on the one hand between the occurrence of allergic disease as perceived by the public, and on the other hand food allergy as can be diagnosed by available clinical and laboratory methods (Altman and Chiaramonte, 1997). The prevalence of food allergy is commonly estimated to be $1-3 \%$ in adults and $6-8 \%$ in children, but the numbers are uncertain (Kanny et al., 2001; Ditto and Grammer, 2002; Sampson, 1999a,b). The relative importance of various food allergens changes rapidly as an individual grows older. Severity of food allergic reactions ranges over a continuous spectrum from trivial symptoms to systemic anaphylactic reactions and death. Regional differences in the prevalence of food allergy may be present, and there certainly are regional differences in Europe with regard to what foods are the most common triggers of food allergic reactions. Risk groups for severe reactions may for this and other reasons also show geographical variation. Norway has about four million inhabitants, and about 20,000 medical doctors. Like everywhere, there is a profound lack of knowledge about the occurrence of food allergy and important aspects such as the incidence of serious food aller- gic reactions, risk groups, risk situations for reactions, specific IgE and skin test positivity for food allergens, incriminating foods, treatment and patient follow-up.

\section{THE NORWEGIAN NATIONAL REPORTING SYSTEM AND REGISTER OF SEVERE ALLERGIC REACTIONS TO FOOD - " FOOD ALLERGY REGISTER 6 ("MATALLERGIREGISTERET")}

The Norwegian National Reporting System and Register of Severe Allergic Reactions to Food was started on $1 \mathrm{Ju} 1 \mathrm{y}, 2000$. It is not a research project, but a permanent information collection system. The first years funding was provided on an annual basis by the Ministry of Health and Social Affairs under its Action Plan Against Asthma, Allergy and Indoor-Air Related Diseases, but the register has later become a permanent institution and is now financed through the ordinary governmental budget. The reporting system represents a collaborative effort between the Norwegian Institute of Public Health that operates the reporting system, and The Norwegian Food Safety Authority (Mattilsynet), with the Norwegian Veterinary Institute as an active partner performing the food allergen analyses. The core of the register is a voluntary reporting system. Submission of a report by a physician requires the 
written consent from patient or guardian. Reporting forms and other materials are mailed once or twice every year to about 6,500 addresses: family doctors, emergency wards, out-patient clinics and relevant hospital departments. The strategy is to reach all relevant first-line care providers. The definition of a severe allergic reaction to food for our purpose had to be simple and pragmatic. A case shall be reported to the Food Allergy Register 'on suspicion', without waiting for further diagnostic work, if a possible food allergyrelated reaction takes place within 24 hours after the intake of a suspected incriminating food, and the reaction was so severe that a physician was contacted within 24 hours because of an emergency or at least for acute help.

\section{PuRPose OF THE NoRWEgIAN NATIONAL REPORTING SYSTEM AND REGISTER OF SEVERE ALLERGIC REACTIONS TO FOOD}

The purposes of the Food Allergy Register are: 1) For health authorities: increased knowledge about the incidence of severe allergic reactions to food in Norway, risk groups, risk situations, geographical distribution, incriminating foods, and how patients are treated and what follow-up is given with regard to advice and care. 2) For food control authorities: food allergen surveillance and safe food for food allergic individuals. That is, to help detect cases in which there are allergens in the food that are not properly declared by labelling or that should not be there according to laws and regulations. 3) For health care providers: increased knowledge about risk groups, risk factors and risk foods, and symptoms and treatment. Increased motivation and attention to the problem of food allergy. Diagnostic support through supplementary specific IgE analyses, food allergen analyses and comments on the food allergy cases. 4) For patients: increased knowledge about risk foods and risk situations, prevention and symptoms. Help to secure safe food for food allergic individuals.

\section{STRUCTURE OF THE REPORTING SYSTEM}

The reporting system is based on:

1. A one-page reporting form to be filled in by the physician, and an informed consent form for the patient to participate in the register and serum bank. Supplementary information may be given on additional sheets or in the form of a copy of the journal entry. The Register is authorised to contact the physician to ask for supplementary information at a later stage when more diagnostic work has been completed. The form asks for patient personal data (personal identification number, name, date of birth, gender, address, regular physician, etc), data for the reporting physician/institution, a brief medical history for the patient with a focus on asthma and allergies, known food allergies, and data on the reported incident: details about the meal(s), patient symptoms, circumstances under which the reaction took place (location, exercise, etc), and treatment.

2. Serum analysis for food allergen-specific IgE: submission of serum is encouraged, but is not obligatory. Serum is analysed by Pharmacia UniCap ${ }^{\square}$ for specific IgE against 12 selected individual food allergens comprising a standard panel. IgE against additional allergens may be tested for as relevant, but as a rule the physician is asked to send blood to a routine laboratory for further diagnostic testing, if required. Further, serum is analysed by an in-house dot-blot technique ('food allergen array') (Wiker et al., 2003), which allows screening for specific IgE to 170 food allergens in one operation. The Register is not supposed to take over the diagnostic work, but to supplement it.

3. When relevant, allergen analysis of the suspected incriminating food is performed by the Norwegian Veterinary Institute. Submission of food for analysis previously should be done only after contact with the local food control authorities. However, as this turned out to be impractical and in effect an obstacle to submitting foods for analysis, the physician now can send in food together with the reporting form and the blood specimen. All laboratory services are offered free of charge.

\section{FEEDBACK FROM THE FOOD ALLERGY REGISTER TO THE REPORTING PHYSICIAN}

Corresponding to the reporting form, serum and food for analysis, physicians submitting a report will receive in return:

1. A comment on the case, with a discussion focused on relevant food allergens and allergies, crossreactions, risk foods, relevant features of symptoms, the relevance of asthma, etc.

2. Results of the serum specific $\operatorname{IgE}$ analysis, with comments (integrated with items 1 and 3). It is always emphasised that serological tests are done only as a support for the diagnosis, and that a diagnosis of food allergy never is made in the laboratory, but in the physician's office. Further, the physicians (and the patients) are warned against eliminating foods from the diet without a good medical reason.

3. Results of the food allergen analysis, when performed. A copy is also sent to the local food control authorities and The Norwegian Food Control Authority (Mattilsynet), so that relevant administrative measures can be taken in case the food has been found to contain allergens not presumed to be there according to the declaration and labelling rules (e.g. milk protein in 'milk-free' products).

\section{RESULTS AND DISCUSSION}

\section{A. Function of the Food Allergy Register}

General acceptance of the Food Allergy Register by patients and physicians

The Norwegian National Reporting System and Register of Severe Allergic Reactions to Food ("Food 
Allergy Register") has been very well received by the patient organisations and the medical profession. Some health institutions have integrated reporting to the Food Allergy Register into their standard procedures. However, a few physicians complain about the additional work and the lack of economic compensation for the time it takes to submit reports. This may be one of the reasons for the incomplete reporting of reportworthy cases (see below). We have no data on how many patients, if any, decline to give their consent for their reaction to be reported. Media are very interested in the Food Allergy Register, which has been featured several times on national TV, radio, and in newspapers and various magazines.

\section{Number of cases reported}

By 1 July 2004, after four years of operation, 300 cases had been reported, that is an average of about 75 cases per year. However, the numbers have increased for every year through 2003 (Fig. 1). The increase probably reflects increasing awareness among physicians and patients of the existence of the Food Allergy Register and its usefulness. Further, the increase in reported cases appears to be an indication that the physicians find the comments on their cases and the results of the serum and food analyses useful, because we see that once a physician has first submitted a report, there is a clear tendency that more reports will come from the same physician or the same clinic. However, we sometimes learn about cases that have not been reported, and there clearly is a large degree of underreporting. One indication of this is that from certain group practices, emergency wards and outpatient clinics we receive a steady trickle of reports, whereas little or nothing is received from other comparable group practices and clinics. Another probable indication of underreporting is the marked geographical differences in number of cases. For example, the Bergen area on the west coast (174,348 households) during the first 18 months reported only three cases, compared to 57 from the Oslo area $(166,506$ households). This difference may, of course, reflect a true difference in incidence of severe food allergic reactions between the Oslo and Bergen areas. However, a more likely explanation is a difference in how often report-worthy reactions are actually reported to the Food Allergy Register from the Oslo area and the Bergen area.

Most information about the prevalence of food allergy and the incidence of severe reactions is based on studies on various patient materials and selected groups. There are only few population-based epidemiological studies (Kanny et al., 2001), and such studies give at best only a snapshot of the situation. Nation-wide registers for some specific allergies exist, namely the American register for peanut and tree nut allergy (Sicherer et al., 2003), but this register is limited to the mentioned specific allergies. Further, its 'catchment area' is limited to members of the Food Allergy and Anaphylaxis Network (about 7,000 lay

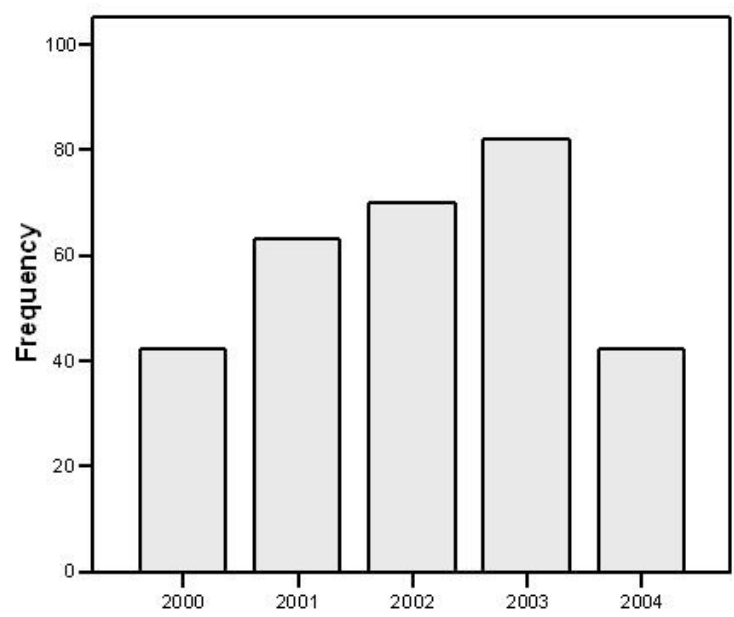

Figure 1. Registrations of severe allergic reactions to food in Norway per year (2000: 6 months, 2004: 6 months).

members and 1,000 health professionals in 1997) and to patients recruited by about 4,000 members of the American Academy of Allergy, Asthma and Immunology. In Sweden, reporting of fatal and life-threatening allergic reactions to food to a central co-ordinator has been encouraged, and all physicians were asked to participate (Foucard and Malmheden-Yman, 1999; 2001). However, this effort was rather loosely organised, and probably more suitable for bringing in illustrative and educational cases than for more comprehensive surveillance. Still, the Swedish effort probably represents the closest parallel to the Norwegian nationwide reporting system and register for severe allergic reactions to food.

The number of cases of severe allergic reactions to food that we could expect if all relevant incidents in Norway were reported to the Food Allergy Register, is difficult to estimate because of the scarcity of data on severe food allergic reactions, and also because of the likeliness of geographical differences. In USA, an annual incidence of food anaphylaxis of 7.6 cases per 100,000 person-years and a food anaphylaxis occurrence rate of 10.8 per 100,000 person-years has been estimated (Yocum et al., 1999). According to this, in Norway we should expect some 400 incidents per year (resulting in 30-40 hospitalisations and 1-2 deaths). With peanuts possibly still representing a more dominating cause for anaphylaxis in USA than in Norway, the estimates for Norway presumably should be somewhat lower compared to the American data. Thus, the reporting rate in Norway (60 to 90 per year so far) may be $1 / 3$ to $1 / 5$ of all cases. The increasing number of reports received by the Food Allergy Register therefore most likely reflects an increase in reporting frequency only, and not an increase in the occurrence of severe food allergic reactions. Clearly, the numbers from the Food Allergy Register are minimum numbers for severe food allergic reactions in Norway (which represent, however, a very useful parameter). Only as the reporting frequency improves, will the numbers get closer to the still unknown true incidence of severe 
food allergic reactions in this country. Also, the value of the data from the Food Allergy Register will increase as the reporting becomes more complete. However, with many reports received from some large, general emergency wards and from physicians all over the country, the data from the Register probably already are fairly representative for the situation in Norway.

\section{Serum samples}

For some $80 \%$ of cases serum has been submitted. An increasing trend for serum submission has been noted, probably reflecting that the physicians find the results of the serological testing useful. Serum is also very useful when testing the food for allergens to which the patient reacts serologically. The UniCap ${ }^{\square}$ panel helps in diagnosis and gives valuable statistical information for some key food allergens. The 170-allergen food allergen array has been found very useful for a wide screening and has helped find the offending allergen in a number of cases. It has sometimes turned out to be a valuable supplement even for allergens included in the UniCap $^{\square}$ panel. Serum samples can be used only to solve problems related to the particular reported case, and not for independent research purposes, for which a special permission is required.

\section{Food allergen analyses}

In far fewer cases than for serum, food has been sent in for food allergen analysis. However, the number is improving. Food allergen analysis has proven to be of great value. A number of cases of allergen contamination or deficient labelling (to be reported in detail elsewhere) have been revealed, for example milk protein-containing hot-dogs sold as 'milk-free', chocolate candy containing milk not indicated on the label, fish cakes contaminated by shellfish allergen (Fæste et al., 2003), and others.

Perhaps the case best illustrating the value of the Food Allergy Register with systematic registration of severe food allergic reactions so that a more comprehensive picture of the situation can be achieved, is the addition of lupine flour to bread and other bakery products. In France, it has for a number of years now been permitted to add up to $10 \%$ lupine flour to wheat flour. It has been reported that there is a clinically relevant cross-reactivity between peanut and lupine, so that anaphylactic reactions may be triggered if a peanut-allergic individual ingests a food containing lupine (Moneret-Vautrin et al., 1999). The Food Allergy Register first received a report on a peanut-allergic individual who experienced repeated reactions to hotdog bread later found to contain lupine flour (Fæste et al., 2004), and we now over a period of about two years have received a total of seven reports on cases with reactions most likely to lupine. Lupine flour appears to be used in imported bakery products, and also to have been taken into use by some Norwegian bakeries. Some of the reported cases were caused by intended use of lupine flour in the bakery products, other cases by inadvertent contamination by lupine, which was used for other products in the same bakery. Allergic reactions to lupine appears to be a new but significant problem in Norway, and we must expect the problem to increase if measures are not taken to inform peanut-allergic individuals about lupine, to improve labelling, and to avoid contamination of wheat flour by lupine in bakeries.

Food allergen analysis should be performed in all cases when there is a specific suspicion that the incriminating food contains one or a limited number of allergens that could be the offending ones. Even in a situation with no specific suspected allergen initially, it must be kept in mind that food analysis can be of great value if specific IgE analysis later suggests allergy to an allergen. Therefore, in many cases, food should be kept on hold awaiting the results of the $\mathrm{IgE}$ analyses. Rather often, patients are not aware of their food allergy until they experience their first severe food allergic reaction. However, an example of a situation when submission of food for analysis is not expected to be useful, is when a multi-allergic patient has reacted to an oriental stew or a pizza with "everything" in the topping.

\section{Lessons from running a food allergy register}

A major challenge with running a reporting system and register like the one we have started in Norway, is to achieve and maintain a high reporting rate. Frequent postal reminders, and reminders and information in professional journals and public media in our experience are exceedingly important. Finally, it is of crucial importance that the reporting physician gets something back that she/he finds useful, in the form of laboratory analyses and clarifying and educational comments on the case.

\section{B. Some specific results from the Food Allergy Register}

\section{Gender distribution}

The gender distribution of severe reactions in our material shows a 60/40 female over male dominance. This gender difference was not apparent at 18 months (Løvik et al., 2003a,b). Gender differences are well known for allergic diseases (Jarvis and Burney, 1997). In the literature, it is sometimes stated that food allergy is more common among women. This has been explained partly by different health-seeking behaviour in men and women. If so, one would expect to see the gender difference predominantly for milder reactions to food. Severe reactions are more likely to have clear physiological mechanisms and to make emergency medical care necessary regardless of differences in health-seeking behaviour, while milder forms of perceived food allergy perhaps more often have other mechanisms and causes. Our data therefore suggest that either is severe food allergy more common in females than in males, or females having a somewhat different food allergen exposure than men. 


\section{Awareness of allergy}

Not unexpectedly, two-thirds of the reported cases had known allergy of any form, and slightly fewer had known (or perceived) food allergy (18-month data, Løvik et al., 2003b). Food allergy tends to occur in subjects who also have other allergies (Emmett et al., 1999), but not always so. A severe reaction is sometimes the first recognised sign of food allergy. Also, "hidden" allergens are a well-recognised problem in food allergy, and a majority of the reported reactions were experienced during meals at restaurants, parties or in institutions (data not shown). Furthermore, a person may misjudge the situation and knowingly eat food that previously has caused reactions, sometimes because of fear to admit an allergy problem or because of alcohol influence. This may particularly be the case with adolescents and young adults, who surprisingly show up as a marked risk population in our material (Fig. 2).

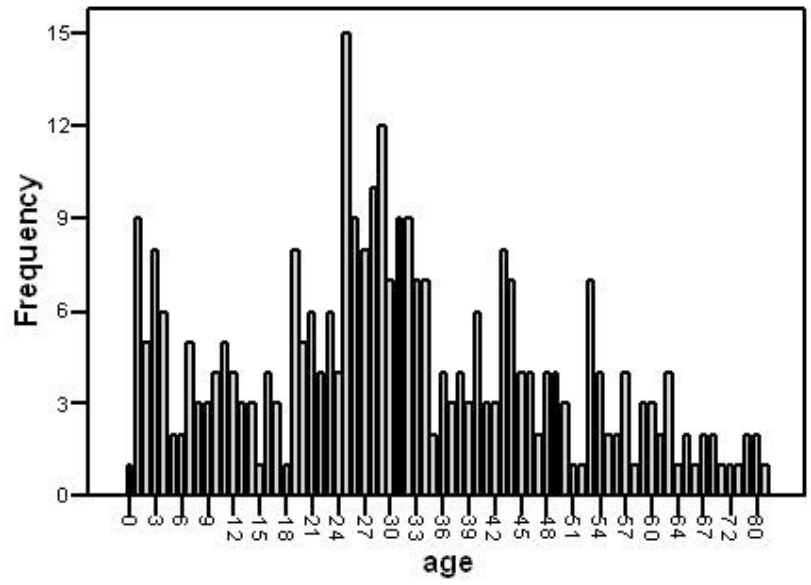

Figure 2. The age distribution of severe allergic reactions to food in Norway shows two peaks: 0-4 year olds and 20-35 year olds.

\section{Age distribution}

The age distribution is shown in Fig. 2. This figure shows one of the most striking pieces of new knowledge obtained from the Food Allergy Register, and it has been consistent since the beginning (Løvik et al., $2003 a, b)$. The main risk group for reported severe allergic reactions to food is comprised of young adults aged twenty to thirty-five. This unexpected finding may have several explanations. Adolescents and young adults probably are the age group in Norway most frequently eating away from home, and they may be the least willing to admit an allergy problem when they are eating out with friends, and alcohol consumption is often high. Our findings with regard to age to some extent resemble those reported for anaphylactic deaths in an American material (Bock et al., 2001), but the American patients on average were in their teens. A newly published report from Great Britain on ana- phylactic deaths is very much in line with our findings with regard to age distribution (Pumphrey, 2004).

\section{Specific IgE and incriminating foods}

There will always be higher numbers for specific IgE than for clinical allergy to a given allergen. The strength of the relationship between seropositivity and clinical food allergy is dependent on the methodology for specific $\operatorname{IgE}$ analysis and the level of specific $\operatorname{IgE}$, and varies for different food allergens (Sampson, 1999a,b, 2001; Sicherer, 2001). However, with these important limitations, the prevalence of specific IgE to food allergens must be assumed to be an indicator of what food allergens are of greatest importance. The highest seroprevalence was found for hazelnut and peanut, and thereafter for celery, shrimp and wheat (data not shown). The high prevalence of specific IgE to peanut may indicate that peanut has become a more important allergen in Norway than hitherto believed, and that the Norwegian population in this aspect resembles the British and US populations. Given that the high frequency of specific IgE to celery is not caused by cross-reactivity, the findings also suggest that celery allergy may be more important than previously thought, and that the Norwegian population in this aspect resembles populations on the European continent.

The data on suspected incriminating foods (not shown) necessarily have considerable uncertainty, but the results generally are in line with the results for specific IgE. The four-year data are not yet ready, but peanut and nuts appeared to be the most important allergens also with regard to clinical disease (Løvik et al., 2003b), and the impression is that this has not changed much since then. Fish allergy appears to be rare.

\section{Time to reaction}

With regard to the time from the suspected causative food intake to beginning of symptoms, about $50 \%$ of the cases experienced symptoms less than 30 minutes after food intake (data not shown). A relationship between seropositivity and time-to-onset of symptoms was observed. Later reactions may more often have other mechanisms than IgE-mediated allergy, or not be hypersensitivity reactions to food at all. A few cases appeared to be food-dependent, exercise-induced reactions (Kidd et al., 1983; Maultiz et al., 1979), which may manifest themselves several hours after food intake.

\section{Treatment of anaphylactic reactions is not optimal}

Our data on treatment (not shown, four-year data in preparation) may indicate that adrenaline is not used as often as recommended for treating serious food allergic reactions. On the other hand, the fact that over $40 \%$ of the cases were given adrenaline alone or together with steroids and antihistamines (18-month data, Løvik et al., 2003b), and that another fourth of the patients were given injected steroids, attests that the 
reactions were judged by the physicians to be severe. For some patients, recommended anaphylaxis treatment was not given because the condition had become much less severe before the patient received medical care. However, the crucial role of injected adrenaline in the treatment of anaphylactic reactions needs to be emphasised.

\section{ACKNOWLEDGEMENTS}

Funding for the first years was provided by the Ministry of Health and Social Affairs under its Action Plan against Asthma, Allergy and Indoor-Air Related Diseases. We thank all physicians and patients who have contributed to the Food Allergy Register.

\section{REFERENCES}

Altman DR, Chiaramonte LT. Public perception of food allergy. Environ Toxicol Pharmacol 1997; 4: 95-99.

Bock SA, Munoz-Furlong A, Sampson HA. Fatalities due to anaphylactic reactions to foods. J Allergy Clin lmmunol 2001; 107: 191-193.

Ditto AM, Grammer LC. Food allergy. In: Grammer LC, Greenberger PA (Eds), Patterson's Allergic Diseases, Lippincott Williams \& Wilkins, Philadelphia, 2002: 257-277.

Emmett SE, Angus FJ, Fry JS, Lee PN. Perceived prevalence of food allergy in Great Britain and its association with other atopic conditions and with peanut allergy in other household members. Allergy 1999; 54: 380-385.

Foucard T, Malmheden Yman I. A study on severe food reactions in Sweden - is soy protein an underestimated cause of food anaphylaxis? Allergy 1999; 54: 261-265.

Foucard T, Malmheden Yman I. Food-induced anaphylaxis. Pediatr Allergy Immunol 2001; 12 (14 Suppl): 97-101.

Fæste CK, Wiker HG, Løvik M, Egaas E. Hidden shellfish allergen in a fish cake. Allergy 2003; 58: 1204-1205.

Fæste CK, Løvik M, Wiker HG, Egaas E. A case of peanut cross-allergy to lupinee flour in a hot dog bread. Int Arch Allergy Immunol 2004; 135: 36-39.

Jarvis D, Burney P. Epidemiology of atopy and atopic diseases. In: Kay AB (Ed), Allergy and Allergic Diseases, Blackwell Science, Oxford, 1997: 1208-1209.

Kanny G, Moneret-Vautrin DA, Flabbee J, Beaudouin E, Morisset M, Thevenin F. Population study of food allergy in France. J Allergy Clin Immunol 2001; 108: 133-140.

Kidd JM, Cohen SH, Sosman AJ, Fink JN. Food-dependent exercise-induced anaphylaxis. J Allergy Clin Immunol 1983; 71: 407-411.

Løvik M, Wiker HG, Stensby BA, Kjelkevik R, Sommer AK, Mangschou B, Omholt-Jensen G, Mork AV, Gondrosen B. The Norwegian National Reporting System and Register of Severe Allergic Reactions to Food. In: Marone G (Ed), Clinical Immunology and Allergy in Medicine. JGC Publishers, Napoli, 2003a: 447-452.

Løvik M, Wiker HG, Kjelkevik R, Stensby BA, Omholt-Jensen G, Guldberg D, Gondrosen B. Severe allergic reactions to food in Norway: Results from the Norwegian National Register of Severe Allergic Reactions to Food. In: Marone G (Ed), Clinical Immunology and Allergy in Medicine. JGC Publishers, Napoli, 2003b: 461-466.

Maulitz RM, Pratt DS, Schocket AL. Exercise-induced anaphylactic reaction to shellfish. J Allergy Clin Immunol 1979; 63: 433-444.

Moneret-Vautrin DA, Guerin L, Kanny G, Flabbee J, Fremont S, Morisset M. Cross-allergenicity of peanut and lupine: the risk of lupine allergy in patients allergic to peanuts. J Allergy Clin Immunol 1999; 104: 883-888.

Pumphrey R, Anaphylaxis: can we tell who is at risk of a fatal reaction? Curr Opin Allergy Clin Immunol 2004; 4: 285-290.

Sampson HA. Utility of food-specific IgE concentrations in predicting symptomatic food allergy. $J$ Allergy Clin Immunol 2001; 107: 891-896.

Sampson HA. Food allergy. 1. Immunopathogenesis and clinical disorders. J Allergy Clin Immunol 1999a; 103: 717-728.

Sampson HA. Food allergy. 2. Diagnosis and management. J Allergy Clin Immunol 1999b; 103: 981-989.

Sicherer SH. Clinical implications of cross-reactive food allergens. J Allergy Clin Immunol 2001; 108: 881-890.

Sicherer SH, Munoz-Furlong A, Sampson HA. Prevalence of peanut and tree nut allergy in the United States determined by means of a random digit dial telephone survey: a 5-year follow-up study. J Allergy Clin Immunol 2003; 112: 1203-1207.

Wiker HG, Stensby BA, Løvik M. Analysis of sera submitted to the Norwegian National Register of Severe Allergic Reactions to Food using a macro-array for food allergy. In: Marone G (Ed), Clinical Immunology and Allergy in Medicine. JGC Publishers, Napoli, 2003: 453-460.

Yocum MW, Butterfield JR, Klein JS, Volcheck GW, Schroeder DR, Silverstein MD. Epidemiology of anaphylaxis in Olmsted County: a population-based study. J Allergy Clin Immunol 1999; 104: 452-456. 